Rev. salud pública. 13 (4): 644-653, 2011

\title{
Asesorías telefónicas en un servicio de toxicología 2006-2009
}

\author{
Toxicology service phone-in assistance, 2006-2009
}

\author{
María F. Olarte-Olarte ${ }^{1,2}$, Ángela F. Espinosa-Aranzales ${ }^{2} y$ \\ Daniel E. Suárez-Acevedo ${ }^{3}$
}

1 Institución Prestadora de Servicios Colsubsidio-Fundación Universitaria para ciencias de la salud
(FUCS). Bogotá, Colombia. franciscaolarte@gmail.com
2 Universidad del Rosario. Bogotá, Colombia. angela.espinosa@urosario.edu.co
3 Universidad de los Andes. Bogotá, Colombia. dsuarezacevedo@yahoo.es

Recibido 6 Diciembre 2010/Enviado para Modificación 20 Mayo 2011/Aceptado 29 Mayo 2011

\section{RESUMEN}

Objetivos Establecer el perfil epidemiológico de las asesorías telefónicas del servicio de toxicología de la IPS Colsubsidio durante los años 2006-2009.

Métodos Estudio descriptivo poblacional de 594 asesorías telefónicas realizadas por el servicio de toxicología a los médicos que atendieron pacientes que consultaron por exposición a sustancias tóxicas.

Resultados En cuanto al grupo etáreo, se presentaron dos picos entre los 0 a los 6 años siendo los hombres los más implicados por causas accidentales y entre 13 a los 18 años en donde predominan las mujeres que se intoxican con fines suicidas. La principal causa de intoxicación fue de origen accidental, seguido por los intentos de suicidio, no encontrando diferencias significativas entre estas proporciones. Los medicamentos son las principales sustancias con las cuales se intoxicó la población del estudio, el tratamiento sintomático fue la terapéutica más usada. Otras variables estudiadas fueron la distribución de las intoxicaciones en el tiempo y la vía de intoxicación.

Conclusiones El perfil de intoxicaciones identificado en esta investigación se asemeja en gran parte a lo reportado en la literatura del resto del continente americano. Llama la atención que no se encontró diferencia estadísticamente significativa entre las intoxicaciones accidentales y las de fines suicidas. Este estudio es un referente estadístico para Bogotá y ciudades similares.

Palabras Clave: Intoxicaciones, asesoramiento, toxicología, suicidio (fuente DeCS, BIREME)

\section{ABSTRACT}

Objective Establishing phone-in assistance's epidemiological profile as part of Colsubsidio's toxicology service (a Colombian healthcare-providing institution -IPS) from 2006-2009. 
Methods This was a population-based descriptive study of 594 toxicology service telephone consultations; it also concerned doctors attending patients seeking advice due to exposure to toxic substances.

Results In terms of exposure to and consumption of toxic substances regarding age group, patients aged 1 day to 6 years reached a peak, males being most affected by accidental causes. Patients aged 13 to 18 also reached a peak, females being most affected due to attempting suicide by deliberately poisoning themselves. The findings revealed that accidental poisoning was the major cause, followed by suicide attempts. There was no significant difference between percentages. Medicaments appeared to be the main substance causing poisoning amongst the study population. Therapeutic treatment was predominantly used. Other variables taken into account in the study included poisoning distribution as time elapsed and poisoning route.

Conclusions The poisoning profile identified in this investigation was comparable (to a large extent) to what has been reported in the specialized literature on the rest of the American continent. It should be stressed that there was no significant statistical difference between accidental poisonings and suicide-related poisoning. This study constitutes a statistical reference for Bogota and similar cities.

Key Words: Poisoning, assistance, toxicology, suicide (source: MeSH, NLM).

$\mathrm{E}$ n la IPS de Colsubsidio hasta antes de 2006 los pacientes intoxicados eran manejados por los servicios de medicina general, pediatría y medicina interna.

El servicio de toxicología clínica de la IPS Colsubsidio, se crea en el 2006, para desarrollar programas de prevención en el consumo de sustancias psicoactivas, atender pacientes que consulten por consumo de las mismas, por exposición tóxica ocupacional, delictiva y para optimizar la atención del paciente intoxicado en los servicios de hospitalización, urgencias y unidad de cuidado intensivo.

También en el 2006, se crea el servicio de asistencia telefónica como una forma de asegurar asesoría permanente a los médicos que la necesiten en la resolución de casos de pacientes que consulten por alguna causa tóxica cuando el especialista no se encuentre en la institución, este servicio funciona las 24 horas del día los 365 días al año.

Desde el segundo semestre de 2006 hasta el primer semestre del 2009, se realizaron un total de 594 asesorías telefónicas las cuales se sistematizaron con el fin de tener las bases para establecer un programa de vigilancia toxicológica que permita conocer los motivos de consulta, las causas de intoxicación, el tratamiento instaurado y la caracterización de la población para desarrollar estrategias de prevención y optimizar los protocolos de atención de pacientes intoxicados en la red de Colsubsidio. 


\section{MATERIALES Y MÉTODOS}

Estudio descriptivo poblacional realizado con base en 594 asesorías telefónicas realizadas por el servicio de toxicología de la IPS Colsubsidio a los médicos que atendieron pacientes que consultaron por exposición a sustancias tóxicas desde julio de 2006 hasta el 30 de junio de 2009.

Se construyó una base de datos en Excel para la recolección de la información. Esta base se iba diligenciando en cada una de las asesorías realizadas, la cual comprendía variables de caracterización poblacional de tipificación de las intoxicaciones, tales como: edad, género, tiempo de distribución de las exposiciones tóxicas, según intencionalidad, tóxico involucrado y vía de exposición.

En el programa de SPSS 17.0 se realizó el análisis univariado, calculando frecuencias absolutas y relativas a las variables cualitativas y medidas de tendencia central y de dispersión a la variable edad, posteriormente ésta se categorizó para analizarla según los grupos etáreos como se encuentran en la literatura.

Se hicieron análisis estratificados por grupo etáreo y por género para los dos principales tipos de intoxicación y se compararon proporciones con el programa de Epi Info versión 6.04d con la aplicación de Epitable.

\section{RESULTADOS}

Desde 01 de julio de 2006 hasta el 30 de junio de 2009 se realizaron un total de 594 asesorías relacionadas con accidentes tóxicos, la Clínica Infantil Colsubsidio fue la institución de la red que más solicitó asesorías con un número de 324 llamadas, que corresponden a un 54,5\% del total, seguida por la Clínica de Colsubsidio Ciudad Roma, la cual solicitó 250 asesorías, que corresponden a un 42,1\% de todas las llamadas, el 3,4\% restante de las asesorías corresponden a llamadas provenientes de otros Centros Médicos de la Red.

La distribución por grupos etáreos permitió observar dos picos. El primero comprendido entre los 0 y los 6 años con un total de 192 asesorías correspondientes al 32,3\% del total y un segundo pico entre los 13 y los 18 años con 139 asesorías correspondientes al 30,1\%. El grupo etáreo que requirió menor asesoramiento fue el de mayores de 70 años con un solo 
caso. El promedio de edad del total de pacientes asesorados es 15,5 años \pm 12,5 con un coeficiente de variación del 80,5\%. Durante el periodo de tiempo mencionado, un $53,5 \%$ de los pacientes asesorados pertenecen al género femenino, con un promedio de edad de 16,0 $\pm 11,9$ con un coeficiente de variación de $74,6 \%$ y el $46,5 \%$ restante pertenecen al género masculino, con un promedio de edad de 14,8 años $\pm 12,9$ y un coeficiente de variación del $87,0 \%$.

El número de asesorías en el segundo semestre de 2006 cuando se estableció el servicio fue de 85 correspondiendo al 14,3\% del total de las asesorías presentadas en este estudio. El último periodo que comprende esta investigación corresponde al primer semestre de 2009 en el que se realizaron 127 asesorías correspondiendo al $21,4 \%$ del total.

El número de asesorías por día, mes y según horario de la recepción de la llamada se puede apreciar en la Tabla 1.

Tabla 1. Asesorías según mes del año, día de la semana y horario de consulta

\begin{tabular}{llllll}
\hline \multicolumn{1}{c}{ Mes } & $\begin{array}{c}\text { Asesorías } \\
(\%)\end{array}$ & $\begin{array}{c}\text { Día de la } \\
\text { semana }\end{array}$ & $\begin{array}{c}\text { Asesorías } \\
(\%)\end{array}$ & Horario & $\begin{array}{c}\text { Asesorías } \\
(\%)\end{array}$ \\
\hline Enero & $60(10,1)$ & Lunes & $91(15,3)$ & Madrugada & $53(8,9)$ \\
Febrero & $52(8,8)$ & & & & \\
Marzo & $53(8,9)$ & Martes & $65(10,9)$ & Mañana & $111(18,7)$ \\
Abril & $54(9,1)$ & & & & \\
Mayo & $60(10,1)$ & Miércoles & $84(14,1)$ & & \\
Junio & $49(8,2)$ & & & & \\
Julio & $54(9,1)$ & Jueves & $73(12,3)$ & Tarde & \\
Agosto & $30(5,1)$ & & & & \\
Septiembre & $40(6,7)$ & Viernes & $76(12,8)$ & & \\
Octubre & $35(5,9)$ & & & Noche & \\
Noviembre & $67(11,3)$ & Sábado & $96(16,2)$ & & \\
Diciembre & $40(6,7)$ & Domingo & $109(18,4)$ & & \\
& & & & & \\
Total & $594(100)$ & Total & $594(100)$ & Total & \\
\hline
\end{tabular}

Respecto al número de asesorías recibidas según el día del mes, no hay ninguna diferencia en la frecuencia de la presentación de las mismas en relación con la primera o la segunda quincena.

Los resultados según la intencionalidad de la exposición tóxica se muestran en la Tabla 2.

Es de resaltar que la diferencia entre las intoxicaciones accidentales y las suicidas no es estadísticamente significativa $(\mathrm{p}=0.307)$ al análisis general; al hacerlo de 
manera estratificada, se encuentran diferencias estadísticamente significativas entre los géneros, siendo mayor las intoxicaciones accidentales en el género masculino $(\mathrm{p}=0.032)$ y mayor las intoxicaciones suicidas en el género femenino $(\mathrm{p}=0.000)$. Así mismo, las intoxicaciones accidentales muestran un comportamiento similar al análisis estratificado por grupos etáreos y género, mientras que las intoxicaciones suicidas en hombres presentan dos picos entre los 13 y los 18 años y entre 25 y los 30 años y en las mujeres el pico relevante es entre los 13 y 18 años.

Tabla 2. Asesorías según intencionalidad

\begin{tabular}{lc}
\hline \multicolumn{1}{c}{ Tipo de intoxicación } & Número de asesorías (\%) \\
\hline Accidental & $231(38,9)$ \\
Delictiva & $56(9,4)$ \\
Laboral & $8(1,3)$ \\
Lúdica & $71(12,0)$ \\
latrogénica & $8(1,3)$ \\
Desconocida & $5(0,8)$ \\
Automedicación & $3(0,5)$ \\
Idiosincrática & $1(0,2)$ \\
Suicida & $211(35,3)$ \\
Total & $594(100,0)$ \\
\hline
\end{tabular}

Los resultados del número de asesorías según grupo de tóxico involucrado, según la ruta de exposición al tóxico y según el tratamiento indicado se encuentran ilustrados en las Tablas 3,4 y 5 respectivamente.

Tabla 3. Asesorías según grupo de tóxico involucrado

\begin{tabular}{|c|c|c|}
\hline Grupo de tóxico & $\begin{array}{c}\text { Número de } \\
\text { Asesorías }\end{array}$ & $\%$ \\
\hline Medicamentos & 278 & 46,8 \\
\hline Plaguicidas & 84 & 14,1 \\
\hline Aseo & 62 & 10,4 \\
\hline Sustancias psicoactivas ilegales & 33 & 5,6 \\
\hline Bebidas energizantes & 30 & 5,1 \\
\hline Medicamentos y bebidas alcohólicas & 18 & 3,0 \\
\hline Desconocida & 15 & 2,5 \\
\hline Gas & 14 & 2,4 \\
\hline Alcohol Tóxico & 13 & 2,2 \\
\hline Sustancias de abuso ilegales y bebidas alcohólicas & 9 & 1,5 \\
\hline Fitoterapéutico & 8 & 1,3 \\
\hline Medicamentos y aseo & 4 & 0,7 \\
\hline Etiología no tóxica & 3 & 0,5 \\
\hline Metales & 3 & 0,5 \\
\hline Plaguicidas y bebidas alcohólicas & 3 & 0,5 \\
\hline Toxinas de origen animal & 3 & 0,5 \\
\hline Hidrocarburos & 2 & 0,3 \\
\hline Sustancias psicoactivas ilegales y medicamentos & 2 & 0,3 \\
\hline Alimentos & 1 & 0,2 \\
\hline Aseo y fitoterapéutico & 1 & 0,2 \\
\hline Bebidas energizantes & 1 & 0,2 \\
\hline Gas y aseo & 1 & 0,2 \\
\hline Medicamentos, aseo y plaguicidas & 1 & 0,2 \\
\hline Plaguicidas y sustancias psicoactivas ilegales & 1 & 0,2 \\
\hline Psicoactivos, medicamentos y bebidas alcohólicas & 1 & 0,2 \\
\hline Total & 594 & 100 \\
\hline
\end{tabular}


Tabla 4. Asesorías según la ruta de exposición al tóxico

\begin{tabular}{lcc}
\hline Ruta de exposición al tóxico & $\begin{array}{c}\text { Número de } \\
\text { asesorías }\end{array}$ & $\%$ \\
\hline Oral & 505 & 85.0 \\
Inhalada & 38 & 6.4 \\
Desconocida & 10 & 1.7 \\
Oral y dérmica & 9 & 1.5 \\
Dérmica & 8 & 1.3 \\
Oral y inhalada & 7 & 1.2 \\
Parenteral & 6 & 1.0 \\
Dérmica y inhalada & 3 & 0.5 \\
Inhalada y ocular & 3 & 0.5 \\
Fumada & 2 & 0.3 \\
Fumada y inhalada & 2 & 0.3 \\
Inhalada y parenteral & 1 & 0.2 \\
Total & 594 & 100.0 \\
\hline
\end{tabular}

Tabla 5. Tratamiento indicado en las asesorías telefónicas

\begin{tabular}{lcc}
\hline \multicolumn{1}{c}{ Procedimiento } & $\begin{array}{c}\text { Número de } \\
\text { asesorías }\end{array}$ & $\%$ \\
\hline Tratamiento sintomático & 322 & 54.2 \\
Descontaminación, tratamiento sintomático & 130 & 21.9 \\
Tratamiento sintomático, antídoto & 45 & 7.6 \\
Descontaminación, tratamiento sintomático, antídoto & 41 & 6.9 \\
No intervención & 31 & 5.2 \\
Observación & 20 & 3.4 \\
Descontaminación & 3 & 0.5 \\
Descontaminación, observación & 2 & 0.3 \\
Total & 594 & 100.0 \\
\hline
\end{tabular}

\section{DISCUSIÓN}

En este estudio, los meses en los cuales se presentaron más intoxicaciones fueron noviembre, mayo y enero, en concordancia, en gran parte, con lo reportado por la literatura (1-4).

La literatura reporta diferencias en cuanto a los meses de mayor demanda de asesorías, para Cisproquim- CIATOX Colombia (5), son mayo y marzo, para CITUC, SERTOX y CIAAT's Argentina (6-8) los meses de mayor demanda de asesorías telefónicas son enero, noviembre y diciembre, que corresponden a los meses de verano.

Según los hallazgos de este estudio, los días de la semana que se solicitaron más asesorías telefónicas fueron los sábados y los domingos, coincidiendo con el estudio de HISPATOX 2006 (9). Según lo reportado por la literatura, durante el fin de semana se tiene más tiempo libre y como consecuencia las personas pueden estar más expuestas a drogas psicoactivas ya sea por celebraciones como forma de cultivar el ocio o como forma de sobre llevar 
la soledad $(10,11)$. Llama la atención lo reportado por el SERTOX 2008 en el cual los días de mayor consulta son los lunes y los miércoles.

El mayor número de asesorías se realizaron durante las horas de la noche y la tarde, datos que coinciden con lo reportado en el CITUC y en el SERTOX.

En este estudio las mujeres comprenden la mayoría de las pacientes con exposición a venenos. En los CIAAT's Argentina (8) y en el NPDS (12) predomina las intoxicaciones en este género a partir de los 15 años de edad, en el SERTOX (7) son los hombres los que más se intoxican y en los estudios de Cisproquim y del Servicio de Toxico vigilancia de la sección de Toxicología Clínica de la Asociación Española de Toxicología $(5,13)$, no reportan diferencia entre géneros.

Se presentaron 2 picos de exposiciones tóxicas relacionadas con los grupos de edad, el primero, entre los 0 y los 6 años en el cual la mayoría de los intoxicados eran hombres, esto se explica ya que los varones en esta edad son más exploradores e inquietos $(12,14)$.

El segundo pico se presenta entre los 13 y los 18 años de edad, esto puede ser explicado porque este tipo de población se encuentra más expuesta al consumo de sustancias psicoactivas y por situaciones de estrés propio de ese ciclo vital $(12,15)$, que pueden terminar en tentativas de suicidio. El género femenino representa la mayoría en este grupo etáreo, lo cual concuerda con la literatura respecto a que las mujeres eligen preferencialmente un método menos agresivo para suicidarse (15). Estos picos de intoxicaciones concuerdan con los referidos en el NPDS, en el SERTOX y en el CITUC y con en el CIAT que reporta la mayor incidencia en personas entre 0 a 14 años. En el Cisproquim el primer grupo etáreo reportado es de los 15 a los 48 años, seguido de los 0 a los 15 años. En este estudio la edad promedio de intoxicación se encuentra en los 15,5 años, en contraposición con el Servicio de Toxico vigilancia de la sección de Toxicología Clínica de la Asociación Española de Toxicología en el cual es de 37,5 años.

Es importante resaltar que en el presente estudio 176 asesorías tuvieron relación con pacientes entre los 19 y los 50 años de edad, población económicamente activa, dato que preocupa por las connotaciones económicas y sociales que esto representa. La mayoría de las exposiciones tóxicas fueron secundarias a causas accidentales, en correspondencia con los hallazgos del NPDS, del Servicio de Toxico vigilancia de la sección de Toxicología Clínica 
de la Asociación Española de Toxicología, del CITUC, del CIAT y del SERTOX y en contraposición a lo reportado por Cisproquim que ubica en primer lugar a los envenenamientos por plaguicidas.

En segundo lugar los hallazgos del presente estudio sitúan a las causas suicidas al igual que a lo reportado en el NPDS y en el CITUC.

Las intoxicaciones de origen iatrogénico ya comienzan a aparecer como causa de consulta en los servicios de urgencias de la Red.

Los medicamentos fueron la primera causa de intoxicación, dentro de este grupo el acetaminofén y los antidepresivos tricíclicos fueron los más involucrados. El NPDS y el CITUC también ubican a los medicamentos en el primer lugar.

El segundo grupo de sustancias que con más frecuencia causaron exposiciones tóxicas fueron los plaguicidas, siendo los inhibidores de la colinesterasa y los anticoagulantes los más implicados. Cisproquim pone a los plaguicidas como primera causa de intoxicación.

En este estudio, de manera preocupante las sustancias psicoactivas ocupan el tercer lugar de consulta.

En cuarto lugar los hallazgos de este estudio sitúan como causa de intoxicación a los elementos de aseo y limpieza, en contraposición con lo referido en NPDS, CITUC, CIAT y SERTOX, que los reportan en segundo lugar.

En 102 asesorías se presentó más de un grupo de tóxicos involucrados.

En este estudio la vía de exposición predominante fue la oral al igual que lo reportado por el NPDS, el CIAT y el SERTOX. Le siguió la vía inhalatoria al igual que el CIAT en contraposición con el NPDS que fue la dérmica y con el SERTOX que la segunda fue la parenteral.

En esta investigación, la mayoría de los pacientes $(54,2 \%)$ recibieron tratamiento sintomático, en segundo lugar se ubicaron los procedimientos de descontaminación más tratamiento sintomático, seguidos del tratamiento sintomático más antídoto y de la descontaminación más tratamiento sintomático más antídoto. Los procedimientos de tratamiento de elección en este estudio se contraponen con el NPDS que ubican la descontaminación 
como primer método de tratamiento y con el SERTOX que en primer lugar de tratamiento ubica a ninguna acción, seguida de hidratación, tratamiento de apoyo y sintomático.

El principal aporte de esta investigación es que recopila información de manera sistemática acerca de la asesoría telefónica prestada a médicos de la IPS Colsubsidio, quienes atienden pacientes intoxicados. Este documento es el primero que se realiza de este tipo en la institución, se convierte en un referente del comportamiento epidemiológico de los envenenamientos de la población bogotana.

Algunas sugerencias que se desprenden de este artículo están relacionadas con la necesidad de implementar un sistema de vigilancia toxicológica que permita sistematizar la información, socializarla y estandarizar acciones y programas de prevención de las intoxicaciones teniendo en cuenta el perfil epidemiológico expuesto, por ejemplo los meses y los días de mayor incidencia de intoxicaciones, los grupos etáreos más expuestos y vulnerables como son los niños y los adolescentes, el envenenamiento como forma de suicidio, el manejo seguro de medicamentos y plaguicidas. Igualmente es importante tener en cuenta para los programas de prevención, el hallazgo referente al consumo de sustancias psicoactivas como tercera causa de asesoría toxicológica.

Teniendo en cuenta el número de asesorías realizadas, se pone de manifiesto la importancia de mantener los programas de educación continua a los profesionales de la salud en las temáticas relacionadas con el manejo de las intoxicaciones.

El perfil de intoxicaciones identificado en esta investigación se asemeja en gran parte a lo reportado en la literatura del resto del continente americano, exceptuando el porcentaje de envenenamientos con fines suicidas, que en el presente estudio fue igualmente prevalente que el de envenenamientos accidentales, por lo tanto es importante tomar acciones encaminadas a la disminución del problema del suicidio •

Conflictos de interés: Ninguno. 


\section{REFERENCIAS}

1. Pérez I, Rodríguez E, Dussán M, Ayala J. Caracterización Psiquiátrica y Social del Intento Suicida Atendido en una Clínica Infantil, 2003-2005. Rev. salud pública (Bogotá), 2007; 9(2): 230-240.

2. Leiva L, Alamos L, Prüssing L. y Uriarte A. Intento de suicidio: características clínicas y epidemiológicas. Sexta Región de Chile 2002-2004. An Pediatr (Barc). 2008; 69(2): 110-144.

3. Campo G, Roa J, Pérez A, Salazar O, Piragauta C, López L, et al. Intento de suicidio en niños menores de 14 años atendidos en el Hospital Universitario del Valle, Cali. Revista Colombia Médica, 2003; 34(1):9-16.

4. López-García A, Serradilla-Rodríguez C, López-Ros S, Navarro-González J. Hospital Universitario Virgen del Rocío, Sevilla. Intentos de suicidio: revisión de 23 casos. Anales Españoles de Pediatría, 2002; 56(Suplemento 5):252-253.

5. Gómez J. Centro de información de seguridad sobre productos químicos. Centro de Intoxicación y Asesoría toxicológica Cisproquim-CIATOX. Colombia Informe de Gestión 2005-2006 Internet. Disponible en: http://www.laseguridad.ws/consejo/ consejo/html/memorias/Memorias_Complementarias_Congreso_39/archivos/ trabajos/medicina/Convenio_Cisproquim_Unal.pdf. Consultado 14 de junio de 2011.

6. Centro de información toxicológica (CITUC), Pontificia Universidad Católica de Chile. Reporte estadístico de 2007 Internet. Disponible en: http://www.cituc.cl/cituc/cituc5.php?a=9. Consultado 14 de junio de 2011.

7. Servicio de Toxicología del Sanatorio de Niños de Rosario Argentina (SERTOX) Informe estadístico de 2008. Internet. Disponible en: http://www.sertox.com.ar/ modules.php? name=Content\&pa=showpage $\&$ pid $=550$. Consultado el 14 de junio de 2011.

8. Ministerio de Salud y Ambiente de la Nación. III Informe Estadístico de Exposiciones a Tóxicos registradas por los CIAAT's (Centros de Información, Asesoramiento y Asistencia Toxicológica) de la República Argentina. 2002 Internet. Disponible en: http://www.msal.gov.ar/redartox/documentos/Reporte2002.pdf Consultado el 14 de junio de 2011.

9. Burillo G, Munné P, Dueñas A, Trujillo M, Jiménez A, Adrián M, et al. HISPATOX 2006. Intoxicaciones agudas: perfil epidemiológico y clínico, y análisis de las técnicas de descontaminación digestiva utilizadas en los servicios de urgencias españoles en el año 2006. Emergencias. 2008; 20:15-26.

10. Moral $\mathrm{M}$, Ovejero A. Análisis diferencial por niveles de edad de las actitudes hacia el consumo de SPA en adolescentes españoles Revista interamericana de psicología. 2005; 39:325-338.

11. Moral M, Ovejero A. Modificación de las actitudes, hábitos y frecuencia de consumo de alcohol y otras sustancias psicoactivas en adolescentes españoles a partir de un programa educativo-preventivo. Revista Colombiana de Psicología, Universidad Nacional de Colombia. 2005; (14):100-118.

12. Bronstein A, Spyker D, Cantillena L, Green J, Rumack B, Heard S. Annual Report of the American Association of Poison Control Center's National Poison Data System (NPDS): 25th Annual Report. Clinical Toxicology. 2007; 46(10):927-1057.

13. Servicio de Toxico vigilancia de la sección de Toxicología Clínica de la Asociación Española de Toxicología. Perfil epidemiológico de las intoxicaciones agudas por productos químicos recogidas en el sistema Español de Toxicovigilancia. En: Net A, MarruecosSant Luis. Intoxicaciones Agudas graves. Barcelona: Ediciones Ars; 2006.

14. Perry H. Special toxicologic considerations in the toddler. In: Erickson T, Ahrens W, Aks S, Baum C, Ling L. Pediatric Toxicology. U.S.A.: Ed Mc Graw Hill; 2004.

15. Woolf A. Special toxicologic considerations in the adolescent. In: Erickson T, Ahrens W, Aks S, Baum C, Ling L. Pediatric Toxicology. U.S.A.: Ed Mc Graw Hill; 2004. 